\title{
Le français langue seconde au Canada
}

\section{Corinne Cordier-Gauthier}

\section{(2) OpenEdition}

Journals

Édition électronique

URL : http://journals.openedition.org/trema/2175

DOI : 10.4000/trema. 2175

ISSN : 2107-0997

\section{Éditeur}

Faculté d'Éducation de l'université de Montpellier

\section{Édition imprimée}

Date de publication : 1 juin 1995

Pagination : 27-37

ISSN : 1167-315X

\section{Référence électronique}

Corinne Cordier-Gauthier, «Le français langue seconde au Canada », Tréma [En ligne], 7 | 1995, mis en ligne le 23 septembre 2013, consulté le 19 avril 2019. URL : http://journals.openedition.org/ trema/2175; DOI : 10.4000/trema.2175

Ce document a été généré automatiquement le 19 avril 2019

Trema 


\title{
Le français langue seconde au Canada
}

\author{
Corinne Cordier-Gauthier
}

\section{Introduction}

1 L'emploi de la dénomination français langue seconde a fait l'objet depuis le début des années 1990 d'un certain nombre de réflexions (Besse, Cuq, Ngalasso, Véronique) sans que l'on soit parvenu à un consensus sur ce qu'elle recouvre exactement. J.-P. Cuq, qui lui consacre tout un manuel, ne parvient pas, au terme de sa réflexion épistémologique, à en proposer une définition englobant la totalité des réalités sociolinguistiques, tant celles-ci sont diverses. Ce flou terminologique nous semble être dû, entre autres, au fait que l'on utilise la même dénomination pour désigner deux réalités à la fois bien différentes et étroitement liées : elle désigne d'un côté la situation géolinguistique et/ou sociopolitique du français, son usage ou son statut dans les divers pays de la francophonie; et de l'autre, la didactique de ce français, que l'on voudrait considérer comme un « sous-ensemble » du français langue étrangère (J.-P. Cuq) et qui se caractériserait par une approche méthodologique spécifique.

2 Le Canada, comme de nombreux autres pays, est composé de communautés linguistiques différentes. La didactique du français comme langue non maternelle s'y est développée de façon spectaculaire durant les trente dernières années et on a assisté à une utilisation de plus en plus fréquente de la dénomination français langue seconde pour désigner toute situation d'enseignement/apprentissage du français comme langue non maternelle. Cet emploi ne semble pas, de ce côté-ci de l'Atlantique, soulever de controverse ni susciter une réflexion épistémologique particulière. Il nous semble cependant important d'intervenir dans ce débat pour tenter d'expliquer ce que l'on entend exactement par français langue seconde au Canada. Qu'en est-il de la dénomination français langue étrangère ? Comment expliquer la tendance actuelle qui est de parler, pratiquement dans tous les cas de figures, de français langue seconde au lieu de français langue étrangère? Les langues en présence, leur statut respectif et les valeurs qu'elles représentent pour les différentes catégories d'apprenants permettent d'apporter une première réponse à ces 
questions. Quelles en sont, au plan didactique, les conséquences et dans quels cadres le français langue seconde est-il enseigné/appris?

\section{Les langues en présence}

3 La question linguistique, qui plonge ses racines dans l'histoire, a pris une ampleur importante depuis les années soixante et elle reste, aujourd'hui encore, au cœur des débats politiques. Le statut de reconnaissance officielle de la langue, et du français en particulier, n'a pas connu au fil des siècles un sort uniforme: depuis « la conquête ", le français se trouve en concurrence avec l'anglais, passant du statut de langue majoritaire au XVIII ${ }^{e}$ siècle à une situation de langue minoritaire aujourd'hui. Son usage, longtemps simple état de fait (avec vide juridique), est maintenant protégé par toute une série de lois et de règlements qui sont encore en évolution (Commissariat aux langues officielles, 1994). Les conséquences qui en découlent sur les perceptions que se font les apprenants (de la langue à enseigner/apprendre) sont donc des facteurs à ne pas négliger. Il n'y a pas lieu ici d'en faire l'étude, mais il faut souligner l'existence de tensions ouvertes ou sousjacentes chaque fois qu'il est question de langue.

\subsection{Statut respectif des deux langues officielles}

4 Pour se faire une idée juste du statut des langues au Canada, il ne faut pas perdre de vue qu'il existe différents paliers institutionnels, le gouvernement fédéral et ceux des provinces, chacun légiférant de façon autonome en matière linguistique.

5 Au plan fédéral, le français, bien que numériquement minoritaire ${ }^{1}$ à l'échelle du pays tout entier, est une des deux langues officielles (la Loi sur les langues officielles est adoptée en 1969). Ce nouveau statut lui permet d'être traité, au plan fédéral, sur un pied d'égalité avec l'anglais. La politique fédérale du bilinguisme tente de promouvoir «l'équité linguistique », comme le rappelle le Commissaire aux langues officielles, chaque Canadien ayant accès à tous les services fédéraux dans la langue de son choix. Il s'agit d'un bilinguisme institutionnel (obligation pour les institutions fédérales d'utiliser le français et l'anglais ainsi que d'offrir leurs services dans les deux langues), ce qui a eu des répercussions importantes en particulier sur le développement des deux langues officielles comme langues "secondes ». Cette politique confère au français, en tant qu' "autre langue officielle», une dimension nouvelle, puisqu'il passe de l'état de fait de langue minoritaire à celui de langue «à statut légal » même si, dans les faits, cela n'est pas tout à fait vrai. Chacune des deux langues officielles devient alors, lorsqu'elle n'est pas langue maternelle, langue seconde.

6 Au plan provincial, l'anglais est la seule langue officielle de huit des dix provinces et des deux territoires formant le nord du pays. Les deux provinces faisant exception sont le Nouveau-Brunswick (officiellement bilingue) et le Québec qui a adopté le français comme seule langue officielle (la Charte de la langue française ou Loi 101 depuis 1977)2. Depuis lors le français est au Québec la langue officielle du travail, de l'administration publique, du monde des affaires et de l'affichage et on a développé la « francisation du Québec »; conséquence importante sur le plan de l'enseignement, les nouveaux immigrants sont tenus de fréquenter les écoles françaises, nouvelle politique qui a fait l'objet de nombreuses polémiques (Taddeo Donat J.). Dans les autres provinces et territoires, le français est la langue maternelle des communautés francophones en situation minoritaire 
${ }^{3}$ qui, à des degrés divers, sont en voie d'assimilation, sauf en Ontario où un certain nombre d'interventions gouvernementales tentent d'endiguer ce mouvement ( $R$. Mougeon).

\subsection{Caractérisation linguistique des locuteurs}

7 Caractériser les locuteurs en fonction de leur connaissance de la ou des langues est un exercice souvent périlleux - la notion même de langue maternelle ${ }^{4}$ n'étant pas nécessairement évidente, surtout dans un pays où les phénomènes de bilinguisme et de transfert linguistique sont en constante évolution ${ }^{5}$ - mais, on distingue habituellement les anglophones, les francophones ${ }^{6}$ et les allophones.

La première langue officielle parlée

\begin{tabular}{lcrrrr} 
& Population totale & anglophones & $\%$ & francophones & \multicolumn{1}{c}{$\%$} \\
CANADA & 26994040 & 19817910 & $(\mathbf{7 4 , 4} \%)$ & 6813220 & $(\mathbf{2 5 , 2} \%)$ \\
Québec & 6810300 & 904300 & $(13,3 \%)$ & 5844435 & $(85,8 \%)$ \\
Ontario & 9977055 & 9282070 & $(93,0 \%)$ & 509650 & $(5,0 \%)$ \\
Nouveau-Brunswick & 716495 & 473415 & $(66,1 \%)$ & 242630 & $(33,9 \%)$ \\
Alberta & 2519185 & 2436270 & $(96,7 \%)$ & 53280 & $(2,0 \%)$ \\
Colombie britannique & 3247505 & 3137105 & $(96,6 \%)$ & 49540 & $(1,5 \%)$ \\
Manitoba & 1079390 & 1018630 & $(94,4 \%)$ & 47800 & $(4,4 \%)$ \\
Nouvelle-Écosse & 890950 & 854095 & $(95,9 \%)$ & 35885 & $(4,0 \%)$ \\
Saskatchewan & 976040 & 950835 & $(97,4 \%)$ & 19805 & $(2,0 \%)$ \\
lle-du-Prince Édouard & 128100 & 122770 & $(95,8 \%)$ & 5280 & $(4,0 \%)$ \\
Terre-Neuve & 563935 & 560540 & $(99,4 \%)$ & 2680 & $(0,5 \%)$ \\
Territoires du N.-O. & 57430 & 51110 & $(89,0 \%)$ & 1390 & $(2,4 \%)$ \\
Yukon & 27655 & 26785 & $(96,9 \%)$ & 845 & $(3,0 \%)$
\end{tabular}

Les langues officielles au Canada, Commissariat aux langues officielles, Statistique Canada.

Le terme francophone désigne l'ensemble des Canadiens d'expression française parlant le français, indépendamment de leur origine ethnique ou de leur langue maternelle; l'immense majorité d'entre eux se trouve au Québec et un peu moins d'un million sont répartis dans l'ensemble du Canada, les plus fortes concentrations se situant en Ontario et au Nouveau-Brunswick.

Le terme allophone désigne les Canadiens dont la langue maternelle n'est ni le français ni l'anglais; il s'agit des immigrants récemment arrivés et de ceux dits de première ou deuxième génération et non encore complètement assimilés. Les langues maternelles de ces immigrants sont désignées par l'expression langue d'origine ». Il est intéressant de noter que, pour qualifier ces langues ${ }^{7}$, ce n'est pas le terme « étrangère » qui a été retenu, ceci dans un souci d'acceptation de la différence allant dans le sens de la politique fédérale de multiculturalisme. Quant aux langues autochtones, on veut les revitaliser ${ }^{8}$; elles deviendraient alors "les premières langues nationales du Canada", et ce, par rapport aux deuxièmes langues nationales, le français et l'anglais (Commissaire aux langues officielles, 1993). 


\subsection{Valeurs respectives du français pour les catégories d'apprenants}

$$
\text { potentiels, selon leur situation sociolinguistique et la région géographique où ils se }
$$
trouvent, le statut d'une langue seconde nécessaire «à échelle variable ». ainsi que langue officielle du Canada - est une véritable langue seconde dans le sens où l'entend $\mathrm{H}$. Besse pour qui il y a «enseignement/apprentissage d'une langue seconde quand ses apprenants ont la possibilité quotidienne d'être confrontés à elle en dehors des cours qui en relèvent» ou encore «de la pratiquer authentiquement». Langue d'intégration à la société francophone québécoise, elle n'en est pas pour autant une langue d'assimilation (ni de survie), l'anglais restant la langue majoritaire du Canada et de l'Amérique du Nord.

Pour les allophones du Québec, surtout concentrés dans la région métropolitaine de Montréal ${ }^{9}$, le français est une langue seconde nécessaire car il est un outil d'intégration à la société québécoise (G. Painchaud). Dans le cas des allophones hors Québec, c'est l'anglais qui est la langue seconde d'intégration et de survie ${ }^{10}$, le français pouvant acquérir parfois pour un petit nombre d'entre eux (par exemple pour des Italiens de la deuxième génération) le statut de "deuxième » langue seconde. L'anglais, devenu la langue d'usage la plus utilisée, parfois la mieux connue (étant la langue d'enseignement), se transforme, par transfert linguistique, en langue "première " au détriment de la langue maternelle (processus d'assimilation). Il faut rappeler que l'anglais continue d'exercer un fort pouvoir d'attraction auprès des allophones et des francophones en situation minoritaire.

Pour les anglophones hors Québec, le français est la langue de l'autre importante communauté, c'est une langue seconde non vitale (on peut fonctionner en anglais seulement, partout au Canada y compris au Québec, l'inverse n'étant pas vrai), mais qui représente un atout humain et professionnel important (la popularité des cours d'immersion auprès de la communauté anglophone en témoigne). Selon son degré de motivation, l'anglophone peut être quotidiennement en contact avec le français par les médias (présents partout), l'étiquetage bilingue ou la recherche des contacts humains dans des zones privilégiées comme celle de la capitale nationale ou les régions à concentration bilingue.

\section{Pertinence des dénominations français langue étrangère et français langue seconde au Canada}

Dans ce contexte sociolinguistique où les questions de langue sont encore aujourd'hui l'objet de débats passionnés, le choix de la dénomination (français langue étrangère ou français langue seconde) n'est pas neutre ni arbitraire. 


\subsection{Inadéquation de la dénomination français langue étrangère}

15 Cette dénomination est, au Canada, de plus en plus ressentie comme tout à fait inadéquate pour caractériser le français à enseigner/apprendre. La place qu'occupe le français, principalement au Québec, est bien différente de celle qui est la sienne dans d'autres pays où il a été «importé»: le français n'est pas la langue d'une lointaine métropole culturelle ou économique, mais une langue nationale, celle d'un des deux peuples fondateurs de la Confédération canadienne. C'est pourquoi il n'est pas, sur le territoire canadien, une langue étrangère, même pour la communauté non francophone hors Québec. On touche là une corde sensible: il s'agit de l'affirmation (pour les francophones) et de la reconnaissance (de la part des anglophones) du fait français au Canada. On ne veut ni ne peut, et pour des raisons parfois différentes, présenter aux apprenants anglophones ou allophones le français comme une langue étrangère. Pour désigner l'autre langue officielle à enseigner/apprendre, il fallait donc trouver un qualificatif autre que la lexie "étrangère " porteuse de connotations potentiellement négatives (refus/acceptation de l'autre comme étranger). Déjà J.P. Vinay faisait remarquer que la dénomination « langue étrangère [ ... ] est impensable pour le français au Canada». Avis que partage aussi P. Dumont: «la dénomination français langue étrangère, si maladroite, si décriée, à juste titre, en particulier par nos collègues québécois.»

Par ailleurs, on est bien loin aujourd'hui de l'époque où l'on utilisait uniquement les méthodes et manuels d'origine européenne qui avaient importé et, du même coup, « imposé » la dénomination français langue étrangère, née en France. Depuis l'introduction, au début des années 1970, de la première méthode à contexte canadien, Dialogue Canada (pour remplacer Voix et images de France largement utilisée jusque là), on a commencé à ne plus présenter aux apprenants le français comme une langue venant d'ailleurs. Ce fut peut-être là le premier coup porté à la légitimité de la dénomination français langue étrangère dans le contexte canadien. L'usage concurrent des deux notions dans le passé et encore parfois sous la plume d'auteurs canadiens, surtout dans des articles de revues d'audience et de diffusion internationales, a pu faire croire à une confusion d'emploi entre les deux dénominations; c'est plutôt, nous semble-t-il, une prise de conscience progressive de l'inadéquation de ce concept dans le contexte canadien qui a conduit à un usage généralisé de la dénomination français langue seconde.

\subsection{Usage et sens de la dénomination français langue seconde}

Dans tous les textes fédéraux et provinciaux, une langue officielle non maternelle est toujours désignée comme "langue seconde». Dans les directives des programmes des différents ministères de l'éducation n'apparait pratiquement jamais la dénomination français langue étrangère, mais bien celle de français langue seconde. Par exemple, le ministère de l'éducation de l'Ontario, dans son Programme d'études commun, distingue deux types principaux d'enseignement du français, celui de la langue maternelle et celui de la langue seconde. Au niveau fédéral, le Commissaire aux langues officielles en fait autant.

18 Il en va de même dans le domaine éditorial et associatif : l'Association des professeurs de français langue seconde ou l'Association québécoise des enseignants et enseignantes de 
français langue seconde le montrent bien par le nom même qu'elles se sont donné et la revue Canadian Modern Language Review, "La revue canadienne des langues vivantes », a pour mandat de «favoriser la diffusion de l'information sur l'enseignement et l'apprentissage des langues secondes ».

19 Ainsi, la dénomination français langue seconde est devenue la seule appellation désormais utilisée pour parler du français comme langue non maternelle à enseigner/apprendre. Dans ces conditions, l'abandon de l'appellation français langue étrangère rend caduque et non pertinente la distinction ou opposition que l'on veut faire ailleurs entre le français langue étrangère et le français langue seconde. Le français langue seconde désigne l'ensemble des situations d'enseignement/apprentissage ainsi que l'ensemble des programmes et approches méthodologiques.

\section{Le français langue seconde au plan didactique}

Une seule désignation ne signifie pas pour autant une uniformité des approches méthodologiques et des programmes qui varient en fonction des institutions, des publics, des objectifs, de la méthodologie et du degré de compétence langagière ou de «bilinguisme" recherchés. Nous nous contenterons de mentionner très brièvement quelques exemples parmi les plus significatifs.

\subsection{L'enseignement du français langue seconde dans les écoles}

21 Au niveau scolaire, il n'y a pas de situation absolument uniforme pour l'ensemble du Canada ; en effet, l'éducation est du ressort des provinces et le statut du français varie localement. En outre, les multiples commissions scolaires qui existent dans chaque province déterminent individuellement leurs propres programmes d'enseignement. Malgré cette diversité, on peut cependant dégager trois grands programmes: le français de base ou programme-cadre de français d'une part et les programmes d'immersion et les classes d'accueil au Québec d'autre part.

Le programme de français de base, offert aux niveaux élémentaire et secondaire, est «la principale méthode d'apprentissage de la langue seconde pour la majorité des élèves » (Commissaire aux langues officielles, 1993). Il se présente sous la forme de périodes quotidiennes d'une durée variant, selon les provinces et les programmes, de 20 à 50 minutes. Ce système a fait l'objet en 1985 d'une étude d'envergure, l'Étude nationale sur les programmes de français de base (M. Duplantié, R. Tremblay). On y recommande une approche intégrée connue sous le nom du Curriculum multidimentionnel (Leblanc, Stern) et qui préconise l'intégration des éléments linguistiques à enseigner en quatre syllabi : langue, culture, communicatif/expérientiel et formation langagière générale. Axé sur la communication et l'expérience, avec l'accent mis sur l'interaction, ce modèle d'enseignement est en train de s'ériger en nonne dans toutes les provinces.

L'immersion, le deuxième type important de programme, ne touche en réalité que $10 \%$ des élèves. Elle se caractérise par le fait que certaines matières (sciences, mathématiques ou géographie) sont enseignées en français à des classes composées essentiellement d'anglophones. On distingue plusieurs types d'immersion (précoce, moyenne, tardive, partielle, totale). Ce modèle d'enseignement a suscité beaucoup d'espoir, mais après 
environ 25 ans d'existence, il fait aujourd'hui l'objet de certaines remises en cause et d'une réflexion critique (C. Germain et P. Calvé).

Au Québec, l'objectif de faire du français la langue commune de tous les Québécois a amené les responsables de l'éducation à créer des classes d'accueil et de francisation pour les élèves issus des communautés culturelles (allophones). On privilégie une « approche fonctionnelle, fondée sur une conception de la langue qui considère celle-ci comme moyen de communication et comme véhicule des réalités socioculturelles d'une collectivité (Gouvernement du Québec, 1985). Parmi les fondements du programme figure une pédagogie qui respecte la culture d'origine de l'élève » (G. Painchaud).

\subsection{L'enseignement du français langue seconde aux adultes}

Nous ne parlerons pas ici de l'enseignement au niveau universitaire, qui est très largement développé mais d'une trop grande diversité (autonomie des universités). Par contre, au plan fédéral, le programme de la formation linguistique est un vaste projet de cours de langue lancé en 1964 par le Bureau des langues visant la «bilinguisation » des employés de la fonction publique fédérale. Rebaptisé aujourd'hui Formation linguistique Canada, ce programme a pour tâche, entre autres, d'élaborer des cours et de dispenser de la formation linguistique "en réponse aux besoins institutionnels des ministères ou organismes fédéraux et en réponse aux aspirations de carrière des fonctionnaires » et s'adresse aux fonctionnaires fédéraux pour leur permettre de « desservir la population canadienne dans la langue officielle de son choix et d'atteindre le niveau de bilinguisme requis par leur poste tout en reconnaissant que les fonctionnaires ont le droit de travailler en anglais ou en français " (Commission de la fonction publique du Canada). Les objectifs visés par les cours et les programmes sont «directement liés aux tâches professionnelles des fonctionnaires [...] et axés sur l'exécution de tâches professionnelles en langue seconde». En français langue seconde, Formation linguistique Canada a récemment (1991) mis sur pied Le Programme de base de français au travail (PBFT) pour les clientèles visant les niveaux A et B (niveaux débutant et intermédiaire, le niveau C étant le niveau dit de bilinguisme reconnu par la fonction publique fédérale), un cours spécialisé pour les cadres de direction, des cours de langue de travail (par exemple Réception téléphonique) et un programme d'immersion en français au Québec, Résibec. Des cours équivalents existent en parallèle pour l'anglais langue seconde.

26 Ces exemples de programmes de français langue seconde sont les plus répandus et témoignent du parti pris d'adapter le mieux possible l'enseignement du français langue seconde aux réalités socioculturelles les plus diverses. Au plan de la réflexion et de la recherche, cette tendance se poursuit; il n'est, pour le confirmer, que de mentionner les deux thèmes choisis pour les deux derniers congrès de l'AQEFLES: "À l'écoute du milieu » en 1994 et «L'enseignement en mutation » en 1995. Il paraît bien difficile, dans ce cas, de vouloir donner une définition du français langue seconde qui soit figée une fois pour toutes dans l'espace et dans le temps.

\section{Conclusion}

27 Le domaine de la didactique du français langue étrangère, le FLE, constitue désormais une discipline établie et autonome tant au plan de la pratique (enseignement/apprentissage) que de la recherche et de la formation. En parlant de FLE, on a posé au départ cette 
dénomination comme un fait accompli, clair et sans équivoque; est-elle aussi nette qu'elle en a l'air? À quelles réalités d'enseignement/apprentissage renvoie-t-elle exactement ? Le fait de désigner ainsi l'enseignement/apprentissage du français comme langue non maternelle, a pu sembler aller de soi tant que l'on a envisagé le français au sein de l'hexagone. Un problème se pose dès que l'on adopte une vision moins ethnocentrique. Comme le fait justement remarquer C. Olivieri dans sa préface du manuel de J.P. Cuq, "la diversité des publics, des conditions et des objectifs d'apprentissage trouve-t-elle sa pleine expression dans ce concept un peu trop réducteur et monosémique du français langue étrangère?» Partant de l'opposition langue maternelle/langue non maternelle, on a un peu rapidement sauté à la conclusion que la langue non maternelle était obligatoirement une langue étrangère. C'est sans doute ne pas tenir compte des réalités hors de France où le français est aussi parlé, enseigné/appris comme une autre langue qui n'est pas nécessairement étrangère dans le pays où elle est parlée/apprise, au Canada par exemple (ou même éventuellement en France, auprès de certaines communautés).

Le débat sur les notions de français langue étrangère/français langue seconde nous amène à nous interroger sur un autre concept, celui de la langue maternelle. Cette dénomination, surtout dans les pays où plusieurs langues sont en contact (bien plus nombreux qu'on ne le croit souvent), n'est pas non plus un concept toujours très clairement défini : quelle est la langue maternelle de l'enfant né d'un mariage linguistiquement mixte et qui apprend simultanément les deux langues de ses parents ? En tant qu'intervenants dans le domaine de la didactique du français, langue non maternelle, seconde ou étrangère, nous devrions nous interroger sur ce que recouvre chacun de ces concepts. En attendant, le Canada semble s'être très bien accommodé de la dénomination français langue seconde puisqu'il l'a adoptée comme la seule pouvant s'inscrire adéquatement dans son contexte socioculturel.

\section{BIBLIOGRAPHIE}

BIBEAU G., « L'immersion : ... De la coupe aux lèvres », in Études de Linguistique Appliquée, n 82, 1991.

CALVÉ P., « Vingt-cinq ans d'immersion au Canada, 1965-1990 », in Études de Linguistique Appliquée, $\mathrm{n}^{\circ}$ 82, 1991.

COMMISSAIRE AUX LANGUES OFFICIELLES, Langue et société, numéro spécial sous la direction de H.H. David Stern : L'enseignement immersif en français, 1984.

COMMISSAIRE AUX LANGUES OFFICIELLES, Rapport annuel 1993.

COMMISSARIAT AUX LANGUES OFFICIELLES, Nos deux langues officielles au fil des ans, édition revue et corrigée, mai 1994.

COMMISSION DE LA FONCTION PUBLIQUE, Approche systémique à la formation linguistique, Ministère des Approvisionnements et Services, Canada, 1989. 
CUQ J.-P., Le français langue seconde. Origines d'une notion et implications didactiques, Paris, Hachette, 1991.

DUMONT P., « Le français langue africaine », in Diagonales n²1, 1992.

DUPLANTIE M. et TREMBLAY R., « Le syllabus communicant/expérientiel, essai de synthèse », in Revue canadienne des langues vivantes, Vol. $47 n^{\circ} 1,1990$.

EDWARDS V., «Études postsecondaires et immersion », in Études de Linguistique Appliquée, n 82, 1991.

GERMAIN C., Évolution de l'enseignement des langues : 5000 ans d'histoire, Paris. CLE International. 1993.

HULLEN J., et LENTZ F., « Pour une rentabilisation des pratiques pédagogiques en immersion », in Études de Linguistique Appliquée, n 82, 1991.

LEBLANC R., « Les programmes d'immersion et l'habileté à communiquer », in Études de Linguistique Appliquée, $\mathrm{n}^{\circ}$ 88, 1992.

LEBLANC R., «Étude nationale sur les programmes de français de base », in Revue canadienne des langues vivantes, Vol. 47, $\mathrm{n}^{\circ}$ 1, octobre 1990.

MAURAIS J., "La francophonie au Québec », in Le français dans l'espace francophone. Description linguistique et sociolinguistique de la francophonie, tome 1 publié sous la direction de Didier de Robillard et Michel Beniamino, Paris, Honoré Champion, 1993.

MOUGEON R., « Le français en Ontario ", in Le français dans l'espace francophone. Description linguistique et sociolinguistique de la francophonie, tome 1 publié sous la direction de Didier de Robillard et Michel Beniamino, Paris, Honoré Champion, 1993.

NGALASSO M. M., « Le concept de français langue seconde », in Études de Linguistique Appliquée, n - 88, 1992.

PAINCHAUD G., «L'enseignement des langues aux jeunes Québécois des communautés culturelles : politiques et programmes ", in Revue de l'ACLA/Journal of the CAAL, vol. 15, n² 2, 1993. REBUFFOT J., L'immersion au Canada, collection Le point sur... Montréal, Centre Éducatif et Culturel, 1992.

SWAIN M. (a), « French Immersion Program in Canada » in Multiculturalism, n 4, 1980. pp. 3-6.

SWAIN M. (b) et LAPKIN S., « Programmes d'immersion au Canada et enseignement aux adultes : existe-t-il un lien? », in Études de Linguistique Appliquée, n 82, 1991.

TADDEO D. J. et TARAS R. C., Le débat linguistique au Québec. La communauté italienne et la langue d'enseignement, Presses Universitaires de Montréal, 1987.

TARDIF C., "Quelques traits distinctifs de la pédagogie d'immersion », in Études de Linguistique Appliquée, $\mathrm{n}^{\circ}$ 82, 1991.

VERONIQUE D., « Langue première, langue seconde, langue étrangère ... » in Le français dans l'espace francophone. Description linguistique et sociolinguistique de la francophonie, tome 1 publié sous la direction de Didier de Robillard et Michel Beniamino, Paris, Honoré Champion, 1993.

VIGNER G., « Français langue seconde, une discipline spécifique » in Diagonales, nº 4, 1987.

VINAY J.-P., « Guy Rondeau », in Études de Linguistique Appliquée, n 75, 1984.

WEBER S., «L'immersion française : comment plonger sans se noyer », in Études de Linguistique Appliquée, n 82, 1991. 


\section{NOTES}

1. Selon le recensement de 1991, $63 \%$ de la population déclare comme langue maternelle l'anglais, $24 \%$ le français et $13 \%$ une langue non officielle, Statistique Canada, catalogue 93-313.

2. L'article 133 de l'Acte de l'Amérique du Nord Britannique (1867), première loi constitutionnelle du Canada, proclamait le bilinguisme institutionnel pour le Parlement du Canada ainsi que celui du Québec.

3. Lors du recensement de 1991, le français a été déclaré comme unique langue maternelle par $4 \%$ de la population hors Québec en tant que réponse unique et par $1 \%$ dans une réponse multiple, Statistique Canada, catalogue 93-313. Au Nouveau-Brunswick, $32 \%$ des répondants déclarent le français comme langue d'usage, Statistique Canada, catalogue 93-317.

4. Dans l'un des deux questionnaires du recensement de 1991 qui portaient sur la langue, il y avait quatre questions distinguant : 1) la langue maternelle qui est « la langue que cette personne a apprise en premier lieu à la maison dans son enfance et qu'elle comprend encore »;2) la langue parlée à la maison « langue que cette personne parle le plus souvent à la maison »;3) la langue officielle connue; et 4) la langue non officielle connue « que cette personne connait assez bien pour soutenir une conversation ".

5. Lors du recensement de 1991, 2,7 \%de la population canadienne (726 000) déclare avoir deux langues maternelles ou plus. Pour ce qui est de la connaissance des langues (toutes langues confondues), $26.3 \%(7,1$ millions) se dit bilingue et 4,4\% (1,2 millions) polyglotte. Mais $16,3 \%$ de la population pouvait soutenir une conversation en anglais et en français ( 4,4 millions). En ce qui concerne le taux de transfert linguistique (lorsque la langue d'usage ou parlée à la maison est différente de la langue maternelle), le taux de transfert du français, langue maternelle, à l'anglais, langue d'usage est très faible au Québec ( $1 \%$ ) mais s'élève à $35 \%$ dans le reste du pays ( $10 \%$ au Nouveau-B., $37 \%$ en Ontario et $50 \%$ ou plus ailleurs), Statistique Canada, catalogues 93-313 et 94-319.

6. Ces termes, qui sont ceux les plus couramment utilisés, n'ont pas toujours une acception très nette et, dans le cas des personnes qui se disent bilingues, ils posent problème. Les chiffres que nous donnons ci-dessous (recensement de 1991) considèrent la première langue officielle parlée (PLOP) : ils combinent de façon objective les diverses données sur la connaissance des langues officielles, la langue maternelle et la langue parlée à la maison. Ces données ne comprennent pas les langues autres que le français et l'anglais. Les langues officielles au Canada, Commissariat aux langues officielles, Statistique Canada.

7. Lors du recensement de 1991, parmi les langues non officielles déclarées comme langue maternelle, sont cités le plus souvent l'italien (512000), le chinois (492000) et l'allemand (476 000), Statistique Canada, catalogue 93-313. Mais si on les considère comme langue d'usage, les chiffres changent quelque peu: le chinois (430 000), l'italien (288 000), le portugais (152000), l'espagnol (145000) et l'allemand (134000) sont parmi les plus communément mentionnées, Statistique Canada, catalogue 93-317.

8. Lors de l'Assemblée des Premières Nations (juin 1993), il a été question de propositions portant sur les « programmes de revitalisation des langues autochtones » avec l'éventuel « octroi d'un statut officiel». Selon le recensement de 1991, 0,7 \% de la population canadienne a déclaré une langue autochtone comme langue maternelle (193 000), Statistique Canada, catalogue 93-313. Ces langues sont déclarées être parlées le plus souvent à la maison par 0,5\% (138 000), Statistique Canada, catalogue 93-317.

9. À Montréal, $70 \%$ de la population a déclaré le français comme langue maternelle, $17 \%$ l'anglais et $17 \%$ une langue non officielle (certains répondants ont indiqué plus d'une langue maternelle). Recensement de 1991, Statistique Canada, catalogue 93-313. 
10. Lors du recensement de 1991, les Canadiens de langue maternelle autre que l'anglais ou le français pouvaient parler l'anglais dans $88 \%$ des cas et le français dans $15 \%$ des cas, Statistique Canada, catalogue 93-318.

\section{RÉSUMÉS}

C'est un ensemble de raisons à la fois historiques, politiques et même méthodologiques qui expliquent l'emploi particulier de la dénomination français langue seconde au Canada. Nous verrons que la dénomination français langue étrangère s'inscrit mal dans le contexte canadien et que les didacticiens l'ont abandonnée pour celle de français langue seconde, celle-ci désignant de façon globale une variété de situations linguistiques et de programmes d'enseignement.

Historical, political and even methodological factors have led to the specific use of the term français langue seconde in Canada. It will be shown that the term français langue seconde is not appropriate in the Canadian context and that it is no longer used in the language-teaching field, having been superseded by français langue seconde, which encompasses a variety of languagerelated situations and of teaching programs.

INDEX

Mots-clés : bilinguisme, langue étrangère, langue officielle, langue seconde

Keywords : bilingualism, foreign language, official language, second language

\section{AUTEUR}

CORINNE CORDIER-GAUTHIER

Carleton University, Ottawa, Canada 